Mathematical Sciences and Applications

E-NOTES

MATHEMATICAL

SCIENCES

AND APPLICATIONS

E-NOTES

https://doi.org/10.36753/mathenot.688493

9 (2) 53-63 (2021) - Research Article

ISSN: 2147-6268

(C)MSAEN

\title{
Jacobi Elliptic Function Solutions of Space-Time Fractional Symmetric Regularized Long Wave Equation
}

\author{
Sevil Çulha Ünal, Ayşegül Daşcıoğlu and Dilek Varol*
}

\begin{abstract}
In this paper, by using a direct method based on the Jacobi elliptic functions, the exact solutions of the space-time fractional symmetric regularized long wave (SRLW) equation have been obtained. The elliptic function solutions of a nonlinear ordinary differential (auxiliary) equation $(d F / d \xi)^{2}=P F^{4}(\xi)+Q F^{2}(\xi)+$ $R$ have also been examined. Besides, the solutions have been found in general form including rational, trigonometric and hyperbolic functions. Moreover, the complex valued solutions, periodic solutions, and soliton solutions, have also been gained. Some solutions have been illustrated by the graphics.
\end{abstract}

Keywords: SRLW equation; Jacobi elliptic function; Nonlinear differential equation; Fractional partial differential equation. AMS Subject Classification (2020): Primary: 33E05, Secondary: 35G20; 35L05; 35R11.

*Corresponding author

\section{Introduction}

The symmetric regularized long wave (SRLW) equation is in the form

$$
u_{t t}+u_{x x}+u u_{x t}+u_{x} u_{t}+u_{x x t t}=0
$$

was first described by Seyler and Fenstermacher [1] in 1984 as a model of the weakly nonlinear ion acoustic and space-charge waves. This equation emerges in various physical applications, such as solitary waves with shallow water waves, ion-acoustic waves in plasma and shallow water waves [2]. The solutions of the SRLW equation has been found by the finite difference method [3], exp-function method [4], $\left(G^{\prime} / G\right)$-expansion method [5], simplest equation method [6], conservative Crank-Nicolson finite difference scheme [7], generalized Jacobi elliptic function method [8], and different version of finite difference method [9].

In recent years, investigation of the exact solutions for fractional differential equations has been popular in the study of scientific research. An important one of these equations is the fractional SRLW equation. So far, the solutions of the space-time fractional SRLW equation has been investigated by utilizing the sub-equation 
method [10], functional variable method [11], exp-function method [11], $\left(G^{\prime} / G\right)$-expansion method [11], tanh-coth method [2], tan-cot method [2], sech-csch method [2] and sec-csc method [2], a novel $\left(G^{\prime} / G\right)$-expansion method [12], Riccati equation method [13], rational $\left(G^{\prime} / G\right)$-expansion method [14], improved $F$-expansion method [15], the extended Jacobi elliptic function expansion method [16], the auxiliary equation method [17], new extended direct algebraic method [18], improved Bernoulli sub-equation function method [19], modified extended tanh method [20], rational $\exp (-\Omega(\eta))$-expansion method [21], $\left(G^{\prime} / G, 1 / G\right)$-expansion method [22], extended auxiliary equation mapping method [23], $\left(D^{\alpha} G / G\right)$-expansion method [24], modified Kudryashov method [25], and the fractional $\left(D_{\xi}^{\alpha} G / G\right)$-expansion method [26]. Among these methods, rational $\left(G^{\prime} / G\right)$-expansion, new extended direct algebraic, improved Bernoulli sub-equation function, and modified extended tanh methods include the conformable derivatives. .

The aim of this paper is to obtain the largest set of exact solutions in the literature of space-time fractional SRLW equation in the form

$$
D_{t}^{\alpha} D_{t}^{\alpha} u+D_{x}^{\beta} D_{x}^{\beta} u+u D_{t}^{\alpha}\left(D_{x}^{\beta} u\right)+\left(D_{t}^{\alpha} u\right)\left(D_{x}^{\beta} u\right)+D_{t}^{\alpha} D_{t}^{\alpha}\left(D_{x}^{\beta} D_{x}^{\beta} u\right)=0,
$$

where $0<\alpha, \beta<1, D_{t}^{\alpha}$ and $D_{x}^{\beta}$ mean conformable fractional derivative of function $u(x, t)$ with respect to $t$ and $x$, respectively.

\section{Preliminaries}

Twelve Jacobi elliptic functions are available in the literature. Basic Jacobi elliptic functions are expressed as

$$
\begin{aligned}
\operatorname{sn} \xi & =\operatorname{sn}(\xi ; m)=\operatorname{sn}\left(\xi \mid m^{2}\right), \\
\operatorname{cn} \xi & =\operatorname{cn}(\xi ; m)=\operatorname{cn}\left(\xi \mid m^{2}\right), \\
\operatorname{dn} \xi & =\operatorname{dn}(\xi ; m)=\operatorname{dn}\left(\xi \mid m^{2}\right)
\end{aligned}
$$

where $m$ is the modulus and is a complex number. When the $m$ is real, it can always be arranged $0<m^{2}<1$. In addition to these functions, Glaisher found the other elliptic functions sd, cd, nd, sc, nc, dc, ns, cs, and ds by taking reciprocals and quotients of basic Jacobi elliptic functions [27]. Besides, when $m=0$ and $m=1$, Jacobi elliptic functions turn into trigonometric and hyperbolic functions [28].

In recent years, Khalil et al. [29] defined a new fractional derivative which is called conformable fractional derivative. This definition is the simplest of other fractional derivatives because it is similar to the definition of the usual derivative. Therefore, the space-time SRLW equation is considered in conformable sense. The definition and the properties of the conformable derivative are given below.

Definition 2.1. [29] Let $f:[0, \infty) \rightarrow R$ be a function. The $\alpha$-th order conformable fractional derivative of $\mathrm{f}$ is defined by

$$
D^{\alpha}(f)(t)=\lim _{\epsilon \rightarrow 0} \frac{f\left(t+\epsilon t^{1-\alpha}\right)-f(t)}{\epsilon}, \quad t>0, \quad \alpha \in(0,1) .
$$

If $f$ is $\alpha$-differentiable in some $(0, \alpha), \alpha>0$ and $\lim _{t \rightarrow 0^{+}} f^{(\alpha)}(t)$ exists, then we define $f^{(\alpha)}(0)=\lim _{t \rightarrow 0^{+}} f^{(\alpha)}(t)$.

Theorem 2.1. [29] Let $\alpha \in(0,1]$ and suppose $f, g$ are $\alpha$-differentiable at point $t>0$. Then, the following are satisfied:

1. $D^{\alpha}(c f+d g)=c D^{\alpha}(f)+d D^{\alpha}(g) \forall c, d \in R$.

2. $D^{\alpha}\left(t^{p}\right)=p t^{p-\alpha} \forall p \in R$.

3. $D^{\alpha}(\lambda)=0$ for all constant functions $f(t)=\lambda$.

4. $D^{\alpha}(f g)=f D^{\alpha}(g)+g D^{\alpha}(f)$.

5. $D^{\alpha}\left(\frac{f}{g}\right)=\frac{g D^{\alpha}(f)-f D^{\alpha}(g)}{g^{2}}$.

6. If, in addition, $f$ is differentiable, then $D^{\alpha}(f)(t)=t^{1-\alpha} \frac{d f}{d t}$. 
Theorem 2.2. [30] Assume $f, g:(0, \infty) \rightarrow R$ be $\alpha$-differentiable functions, where $0<\alpha \leq 1$. Let $h(t)=f(g(t))$. Then $h(t)$ is $\alpha$-differentiable for all $t$ with $t \neq 0$ and $g(t) \neq 0$ we have

$$
D^{\alpha}(h)(t)=D^{\alpha}(f)(g(t)) \cdot D^{\alpha}(g)(t) \cdot g(t)^{\alpha-1} .
$$

If $t=0$ we have

$$
D^{\alpha}(h)(0)=\lim _{t \rightarrow 0} D^{\alpha}(f)(g(t)) \cdot D^{\alpha}(g)(t) \cdot g(t)^{\alpha-1} .
$$

\section{Solutions of the space-time fractional SRLW eqquation}

In this section, utilizing a transformation

$$
\xi=k \frac{t^{\alpha}}{\alpha}+l \frac{x^{\beta}}{\beta}
$$

such that $k$ and $l$ are constants and utilizing the chain rule, space-time fractional SRLW Eq. (1.1) turns into

$$
k^{2} l^{2} \frac{d^{4} u}{d \xi^{4}}+\left(k^{2}+l^{2}\right) \frac{d^{2} u}{d \xi^{2}}+k l u \frac{d^{2} u}{d \xi^{2}}+k l\left(\frac{d u}{d \xi}\right)^{2}=0 .
$$

The main idea of proposed method is to obtain the largest exact solutions of Eq. (3.1) in the form

$$
u(\xi)=\sum_{j=0}^{N} a_{j} F^{j}(\xi) .
$$

Here, $N$ and $a_{j}$ are unknown coefficients. $F(\xi)$ is the solution of nonlinear ordinary differential equation

$$
\left(F^{\prime}\right)^{2}(\xi)=P F^{4}(\xi)+Q F^{2}(\xi)+R
$$

where $P, Q$ and $R$ are constants. This equation has emerged as an auxiliary equation for the solution of many partial differential equations. Differentiating Eq. (3.2), we get the Duffing equation as

\begin{tabular}{|c|c|c|c|c|}
\hline & $P$ & $Q$ & $R$ & $F$ \\
\hline 1 & $m^{2}$ & $-\left(1+m^{2}\right)$ & 1 & $\pm \operatorname{sn} \xi, \quad \pm \operatorname{cd} \xi$ \\
\hline 2 & 1 & $-\left(1+m^{2}\right)$ & $m^{2}$ & $\pm \mathrm{ns} \xi, \pm \mathrm{dc} \xi$ \\
\hline 3 & $-m^{2}$ & $-\left(1+m^{2}\right)$ & -1 & $\pm i s n \xi, \quad \pm i c d \xi$ \\
\hline 4 & -1 & $-\left(1+m^{2}\right)$ & $-m^{2}$ & \pm ins $\xi, \quad \pm i \mathrm{dc} \xi$ \\
\hline 5 & 1 & $2-m^{2}$ & $1-m^{2}$ & $\pm \operatorname{cs} \xi, \quad \pm i d n \xi$ \\
\hline 6 & $1-m^{2}$ & $2-m^{2}$ & 1 & $\pm \operatorname{sc} \xi, \quad \pm$ ind $\xi$ \\
\hline 7 & 1 & $2 m^{2}$ & $m^{2} \quad 1$ & $1 i c s \zeta, \quad 1 \operatorname{dn} \zeta$ \\
\hline 8 & $m^{2}-1$ & $2-m^{2}$ & -1 & $\pm i s c \xi, \quad \pm \mathrm{nd} \xi$ \\
\hline 9 & $1-m^{2}$ & $2 m^{2}-1$ & $-m^{2}$ & $\pm \mathrm{nc} \xi, \quad \pm i m s \mathrm{~d} \xi$ \\
\hline 10 & $-m^{2}$ & $2 m^{2}-1$ & $1-m^{2}$ & $\pm \operatorname{cn} \xi, \quad \pm \frac{i}{m} \mathrm{ds} \xi$ \\
\hline 11 & $m^{2}-1$ & $2 m^{2}-1$ & $m^{2}$ & \pm inc $\xi, \quad \pm m s \mathrm{~d} \xi$ \\
\hline 12 & $m^{2}$ & $2 m^{2}-1$ & $m^{2}-1$ & $\pm i \mathrm{en} \xi, \quad \pm \frac{1}{m} \mathrm{~d} \varepsilon \xi$ \\
\hline 13 & $m^{4}-m^{2}$ & $2 m^{2}-1$ & 1 & $\pm \frac{t}{m} \mathrm{nc} \xi, \pm \mathrm{sd} \xi$ \\
\hline 14 & 1 & $2 m^{2}-1$ & $m^{4}-m^{2}$ & $\pm i m \operatorname{cn} \xi, \pm \mathrm{d} s \xi$ \\
\hline
\end{tabular}

$$
F^{\prime \prime}(\xi)=2 P F^{3}(\xi)+Q F(\xi)
$$

which is used as a mathematical model of various physical systems [31]. The authors are investigated the exact solutions of Eq. (3.2). Some of them are seen in Ref. [32-36]. The Jacobi elliptic function solutions of Eq. (3.2) are presented by Table 1 .

Table 1. The Jacobi elliptic function solutions of Eq. (3.2). 


\begin{tabular}{|c|c|c|c|c|}
\hline 15 & $-m^{4}+m^{2}$ & $2 m^{2}-1$ & -1 & $\pm \frac{1}{m} \mathrm{nc} \xi, \pm i s \mathrm{~d} \xi$ \\
\hline 16 & -1 & $2 m^{2}-1$ & $-m^{4}+m^{2}$ & $\pm m \mathrm{cn} \xi, \quad \pm i \mathrm{ds} \xi$ \\
\hline 17 & $\frac{1}{4}$ & $\frac{1+m^{2}}{2}$ & $\frac{\left(1-m^{2}\right)^{2}}{4}$ & $\begin{array}{cl}\mathrm{ds} \xi \pm \operatorname{cs} \xi, & -\mathrm{d} s \xi \mp \operatorname{cs} \xi \\
i(m \mathrm{cn} \xi \pm \operatorname{dn} \xi), & -i(m \operatorname{cn} \xi \pm \mathrm{dn} \xi)\end{array}$ \\
\hline 18 & $\frac{\left(1-m^{2}\right)^{2}}{4}$ & $\frac{1+m^{2}}{2}$ & $\frac{1}{4}$ & $\begin{array}{l}\frac{\operatorname{sn} \xi}{\operatorname{dn} \xi \pm \operatorname{cn} \xi}, \frac{-\operatorname{sn} \xi}{\operatorname{dn} \xi \pm \operatorname{cn} \xi^{\prime}} \\
\frac{i}{m \operatorname{cn} \xi \pm \operatorname{dn} \xi}, \frac{-i}{m \operatorname{cn} \xi \pm \operatorname{dn} \xi}\end{array}$ \\
\hline 19 & $-\frac{1}{4}$ & $\frac{1+m^{2}}{2}$ & $-\frac{\left(1-m^{2}\right)^{2}}{4}$ & $\begin{array}{l}i(\mathrm{ds} \xi \pm \operatorname{cs} \xi),-i(\mathrm{ds} \xi \pm \operatorname{cs} \xi) \\
m \operatorname{cn} \xi \pm \operatorname{dn} \xi,-m \mathrm{cn} \xi \mp \operatorname{dn} \xi\end{array}$ \\
\hline 20 & $\frac{\left(1-m^{2}\right)^{2}}{4}$ & $\frac{1+m^{2}}{2}$ & $-\frac{1}{4}$ & $\begin{array}{l}\frac{i \operatorname{sn} \xi}{\operatorname{dn} \xi \pm \operatorname{cn} \xi}, \frac{-i \operatorname{sn} \xi}{\operatorname{dn} \xi \pm \operatorname{cn} \xi^{\prime}} \\
\frac{1}{m \operatorname{cn} \xi \pm \operatorname{dn} \xi}, \frac{-1}{m \operatorname{cn} \xi \pm \operatorname{dn} \xi}\end{array}$ \\
\hline 21 & $\frac{1-m^{2}}{1}$ & $\frac{1+m^{2}}{2}$ & $\frac{1-m^{2}}{1}$ & $\begin{array}{c}\mathrm{nc} \xi \pm \mathrm{sc} \xi,-\mathrm{nc} \xi \mp \mathrm{sc} \xi \\
i(m \mathrm{dd} \xi \pm \mathrm{nd} \xi),-i(\operatorname{msd} \xi \pm \mathrm{nd} \xi) .\end{array}$ \\
\hline 22 & $\frac{m^{2}-1}{4}$ & $\frac{1+m^{2}}{2}$ & $\frac{m^{2}-1}{4}$ & $\begin{array}{l}i(\mathrm{nc} \xi \pm \mathrm{sc} \xi),-i(\mathrm{nc} \xi \pm \mathrm{sc} \xi) \\
m \mathrm{sd} \xi \pm \mathrm{nd} \xi,-m \mathrm{sd} \xi \mp \mathrm{nd} \xi\end{array}$ \\
\hline 23 & $\frac{1}{4}$ & $\frac{m^{2}-2}{2}$ & $\frac{m^{4}}{4}$ & $\begin{array}{c}\mathrm{ns} \xi \pm \mathrm{d} s \xi,-\mathrm{ns} \xi \mp \mathrm{ds} \xi \\
\mathrm{dc} \xi \pm \sqrt{1-m^{2}} \mathrm{nc} \xi,-\mathrm{dc} \xi \mp \sqrt{1-m^{2}} \mathrm{nc} \xi\end{array}$ \\
\hline 24 & $\frac{m^{4}}{4}$ & $\frac{m^{2}-2}{2}$ & $\frac{1}{4}$ & $\begin{array}{c}\frac{\operatorname{sn} \xi}{1 \pm \operatorname{dn} \xi}, \frac{-\operatorname{sn} \xi}{1 \pm \operatorname{dn} \xi^{\prime}} \\
\frac{\operatorname{cn} \xi}{\operatorname{dn} \xi \pm \sqrt{1-m^{2}}}, \frac{-\mathrm{cn} \xi}{\operatorname{dn} \xi \pm \sqrt{1-m^{2}}}\end{array}$ \\
\hline 25 & $-\frac{1}{4}$ & $\frac{m^{2}-2}{2}$ & $-\frac{m^{4}}{4}$ & $\begin{array}{c}i(\mathrm{~ns} \xi \pm \mathrm{d} s \xi),-i(\mathrm{~ns} \xi \pm \mathrm{d} s \xi) \\
i\left(\mathrm{dc} \xi \pm \sqrt{1-m^{2}} \mathrm{nc} \xi\right),-i\left(\operatorname{dc} \xi \pm \sqrt{1-m^{2}} \mathrm{nc} \xi\right)\end{array}$ \\
\hline 26 & $-\frac{m^{4}}{4}$ & $\frac{m^{2}-2}{2}$ & $-\frac{1}{4}$ & $\begin{array}{c}\frac{i \operatorname{sn} \zeta}{1 \pm \operatorname{dn} \xi}, \frac{-i \operatorname{sn} \zeta}{1 \pm \operatorname{dn} \xi} \\
\frac{i \operatorname{cn} \xi}{\operatorname{dn} \xi \pm \sqrt{1-m^{2}}}, \frac{-i \operatorname{cn} \xi}{\operatorname{dn} \xi \pm \sqrt{1-m^{2}}}\end{array}$ \\
\hline 27 & $\frac{m^{2}}{4}$ & $\frac{m^{2}-2}{2}$ & $\frac{m^{2}}{4}$ & $\begin{array}{c}\operatorname{sn} \xi \pm i c n \xi,-\operatorname{sn} \xi \bar{\mp} i \mathrm{cn} \xi \\
\operatorname{cd} \xi \pm i \sqrt{1-m^{2}} \mathrm{~s} \xi,-\operatorname{cd} \xi \mp i \sqrt{1-m^{2}} \mathrm{sd} \xi\end{array}$ \\
\hline 28 & $-\frac{m^{2}}{4}$ & $\frac{m^{2}-2}{2}$ & $-\frac{m^{2}}{4}$ & $\begin{array}{c}\mathrm{cn} \xi \pm i \mathrm{sn} \xi,-\operatorname{cn} \xi \mp i \operatorname{sn} \xi \\
\sqrt{1-m^{2}} \mathrm{sd} \xi \pm i \mathrm{~cd} \xi,-\sqrt{1-m^{2}} \mathrm{sd} \xi \mp i \mathrm{~cd} \xi\end{array}$ \\
\hline 29 & $\frac{1}{4}$ & $\frac{1-2 m^{2}}{2}$ & $\frac{1}{4}$ & $\begin{array}{c}\mathrm{ns} \xi \pm \operatorname{cs} \xi,-\mathrm{ns} \xi \mp \mathrm{cs} \xi \\
m \operatorname{sn} \xi \pm i \operatorname{dn} \xi,-m \operatorname{sn} \xi \mp i \operatorname{dn} \xi \\
\operatorname{dc} \xi \pm \sqrt{1-m^{2}} \mathrm{sc} \xi,-\operatorname{dc} \xi \mp \sqrt{1-m^{2}} \operatorname{sc} \xi \\
\operatorname{mcd} \xi \pm i \sqrt{1-m^{2}} \mathrm{nd} \xi,-m \operatorname{cd} \xi \mp i \sqrt{1-m^{2}} \mathrm{nd} \xi\end{array}$ \\
\hline 30 & $-\frac{1}{4}$ & $\frac{1-2 m^{2}}{2}$ & $-\frac{1}{4}$ & $\begin{array}{c}i(\mathrm{~ns} \xi \pm \mathrm{cs} \xi),-i(\mathrm{~ns} \xi \mp \mathrm{cs} \xi), \\
\operatorname{dn} \xi \pm i m s \mathrm{n} \xi,-\operatorname{dn} \xi \mp i m s \mathrm{n} \xi, \\
i\left(\mathrm{dc} \xi \pm \sqrt{1-m^{2}} \operatorname{sc} \xi\right),-i\left(\operatorname{dc} \xi \pm \sqrt{1-m^{2}} \mathrm{sc} \xi\right) \\
\sqrt{1-m^{2}} \mathrm{nd} \xi \pm i m c \mathrm{~d} \xi,-\sqrt{1-m^{2}} \mathrm{nd} \xi \mp i m c \mathrm{~d} \xi\end{array}$ \\
\hline
\end{tabular}

Balancing the highest order linear term

$$
O\left(\frac{d^{4} u}{d \xi^{4}}\right)=N+4
$$

and the highest order nonlinear term

$$
O\left(u \frac{d^{2} u}{d \xi^{2}}\right)=2 N+2
$$

$N=2$ is obtained. Therefore, the solution of Eq. (3.1) can be given as

$$
u(\xi)=\sum_{j=0}^{2} a_{j} F^{j}(\xi)=a_{0}+a_{1} F+a_{2} F^{2} .
$$


Differentiating this equation four times and then substituting the derivatives into Eq. (3.1), sixth order polynomial in $F$ is obtained. Setting its coefficients to be zero, the following equations system is gained,

$$
\begin{gathered}
8 k^{2} l^{2} R Q a_{2}+2\left(k^{2}+l^{2}\right) R a_{2}+2 k l R a_{0} a_{2}+k l R a_{1}^{2}=0 \\
k^{2} l^{2} Q^{2} a_{1}+12 k^{2} l^{2} P R a_{1}+\left(k^{2}+l^{2}\right) Q a_{1}+k l a_{0} Q a_{1}+6 k l R a_{1} a_{2}=0 \\
16 k^{2} l^{2} Q^{2} a_{2}+72 k^{2} l^{2} P R a_{2}+4\left(k^{2}+l^{2}\right) Q a_{2}+4 k l Q a_{0} a_{2}+2 k l Q a_{1}^{2}+6 k l R a_{2}^{2}=0 \\
20 k^{2} l^{2} P Q a_{1}+2\left(k^{2}+l^{2}\right) P a_{1}+2 k l P a_{0} a_{1}+9 k l Q a_{1} a_{2}=0 \\
120 k^{2} l^{2} Q P a_{2}+6\left(k^{2}+l^{2}\right) P a_{2}+6 k l P a_{0} a_{2}+3 k l P a_{1}^{2}+8 k l Q a_{2}^{2}=0 \\
24 k^{2} l^{2} P^{2} a_{1}+12 k l P a_{1} a_{2}=0 \\
120 k^{2} l^{2} P^{2} a_{2}+10 k l P a_{2}^{2}=0 .
\end{gathered}
$$

Solving this nonlinear system, the unknown coefficients are found

$$
a_{0}=B+4 Q A, \quad a_{1}=0, \quad a_{2}=12 P A
$$

\begin{tabular}{|c|c|}
\hline 1,3 & $\begin{array}{l}u=B-4 A\left(1+m^{2}\right)+12 A m^{2} \operatorname{sn}^{2} \xi \\
u=B-4 A\left(1+m^{2}\right)+12 A m^{2} \operatorname{cd}^{2} \xi\end{array}$ \\
\hline 2,4 & $\begin{array}{l}u=B-4 A\left(1+m^{2}\right)+12 A \mathrm{~ns}^{2} \xi \\
u=B-4 A\left(1+m^{2}\right)+12 A \mathrm{dc}^{2} \xi\end{array}$ \\
\hline 5,7 & $\begin{array}{l}u=B+4 A\left(2-m^{2}\right)+12 A \operatorname{cs}^{2} \xi \\
u=B+4 A\left(2-m^{2}\right)-12 A \operatorname{dn}^{2} \xi\end{array}$ \\
\hline 6,8 & $\begin{array}{l}u=B+4 A\left(2-m^{2}\right)+12 A\left(1-m^{2}\right) \operatorname{sc}^{2} \xi \\
u=B+4 A\left(2-m^{2}\right)-12 A\left(1-m^{2}\right) \mathrm{nd}^{2} \xi\end{array}$ \\
\hline $\begin{array}{l}9,11 \\
13,15\end{array}$ & $\begin{array}{l}u=B+4 A\left(2 m^{2}-1\right)+12 A\left(1-m^{2}\right) \mathrm{nc}^{2} \xi \\
u=B+4 A\left(2 m^{2}-1\right)+12 A\left(-m^{2}+m^{4}\right) \operatorname{sd}^{2} \xi\end{array}$ \\
\hline $\begin{array}{l}10,12 \\
14,16\end{array}$ & $\begin{array}{l}u=B+4 A\left(2 m^{2}-1\right)-12 A m^{2} \mathrm{cn}^{2} \xi \\
u=B+4 A\left(2 m^{2}-1\right)+12 A \mathrm{ds}^{2} \xi\end{array}$ \\
\hline
\end{tabular}

such that $A=-k l, B=-\left(k^{2}+l^{2}\right) /(k l)$. Hence, the solution of the Eq. (3.1) is

$$
u=B+4 Q A+12 P A F^{2} .
$$

Substituting the P, Q and F given in Table 1 into expression (3.3), exact solutions of Eq. (3.1) are gained and also illustrated by Table 2. Besides, the solutions of space-time fractional Eq. (1.1) can be also obtained by taking inverse transformation.

Table 2. The Jacobi elliptic function solutions of Eq. (3.2). 


\begin{tabular}{|c|c|}
\hline 17,19 & $\begin{array}{l}u=B+2 A\left(1+m^{2}\right)+3 A(\mathrm{ds} \xi \mp \mathrm{cs} \xi)^{2}, \\
u=B+2 A\left(1+m^{2}\right)-3 A(m \mathrm{cn} \xi \mp \operatorname{dn} \xi)^{2}\end{array}$ \\
\hline 18,20 & $\begin{array}{l}u=B+2 A\left(1+m^{2}\right)+3 A\left(1-m^{2}\right)^{2} \operatorname{sn}^{2} \xi /(\operatorname{dn} \xi \mp \mathrm{cn} \xi)^{2} \\
u=B+2 A\left(1+m^{2}\right)-3 A\left(1-m^{2}\right)^{2} /(m \mathrm{cn} \xi \mp \operatorname{dn} \xi)^{2}\end{array}$ \\
\hline 21,22 & $\begin{array}{l}u=B+2 A\left(1+m^{2}\right)+3 A\left(1-m^{2}\right)(\mathrm{nc} \xi \mp \mathrm{sc} \xi)^{2}, \\
u=B+2 A\left(1+m^{2}\right)-3 A\left(1-m^{2}\right)(m s \mathrm{~d} \xi \mp \mathrm{nd} \xi)^{2}\end{array}$ \\
\hline 23,25 & $\begin{array}{l}u=B+2 A\left(m^{2}-2\right)+3 A(\mathrm{~ns} \xi \mp \mathrm{ds} \xi)^{2}, \\
u=B+2 A\left(m^{2}-2\right)+3 A\left(\mathrm{dc} \xi \mp \sqrt{1-m^{2}} \mathrm{nc} \xi\right)^{2}\end{array}$ \\
\hline 24,26 & $\begin{array}{l}u=B+2 A\left(m^{2}-2\right)+3 A m^{4} \operatorname{sn}^{2} \xi /(1 \mp \operatorname{dn} \xi)^{2}, \\
u=B+2 A\left(m^{2}-2\right)+3 A m^{4} \operatorname{cn}^{2} \xi /\left(\sqrt{1-m^{2}} \mp \operatorname{dn} \xi\right)^{2}\end{array}$ \\
\hline 27,28 & $\begin{array}{l}u=B+2 A\left(m^{2}-2\right)+3 A m^{2}(\operatorname{sn} \xi \mp i \mathrm{cn} \xi)^{2} \\
u=B+2 A\left(m^{2}-2\right)+3 A m^{2}\left(\operatorname{cd} \xi \mp i \sqrt{1-m^{2}} \operatorname{sd} \xi\right)^{2}\end{array}$ \\
\hline 29,30 & $\begin{array}{l}u=B+2 A\left(1-2 m^{2}\right)+3 A(\mathrm{~ns} \xi \mp \operatorname{cs} \xi)^{2} \\
u=B+2 A\left(1-2 m^{2}\right)+3 A(m \operatorname{sn} \xi \mp i \operatorname{dn} \xi)^{2} \\
u=B+2 A\left(1-2 m^{2}\right)+3 A\left(\operatorname{dc} \xi \mp \sqrt{1-m^{2}} \operatorname{sc} \xi\right)^{2} \\
u=B+2 A\left(1-2 m^{2}\right)+3 A\left(m \operatorname{cd} \xi \mp i \sqrt{1-m^{2}} \mathrm{nd} \xi\right)^{2}\end{array}$ \\
\hline
\end{tabular}

The elementary function solutions of Eq. (3.1) are also obtained in Table 3 by using the solutions in Table 2 and the Jacobi elliptic functions for $m=0$ and $m=1$.

Table 3: The elementary function solutions of Eq. (3.2).

\begin{tabular}{|l|l|}
\hline \multicolumn{1}{|c|}{$\boldsymbol{m}=\mathbf{0}$} & \multicolumn{1}{c|}{$\boldsymbol{m}=\mathbf{1}$} \\
\hline$u=B-A$, & $u=B+A$, \\
$u=B-4 A$, & $u=B-8 A+12 A \tanh ^{2} \xi$, \\
$u=B+8 A+12 A \tan ^{2} \xi$, & $u=B-8 A+12 A \operatorname{coth}^{2} \xi$, \\
$u=B+8 A+12 A \cot ^{2} \xi$, & $u=B+4 A-12 A \operatorname{sech}^{2} \xi$, \\
$u=B-4 A+12 A \sec ^{2} \xi$, & $u=B+4 A+12 A \operatorname{csch}^{2} \xi$, \\
$u=B-4 A+12 A \csc ^{2} \xi$, & $u=B-2 A+3 A(\tanh \xi \pm i \operatorname{sech} \xi)^{2}$, \\
$u=B+2 A+3 A\left(\sec ^{2} \pm \tan \xi\right)^{2}$, & $u=B-2 A+3 A(\operatorname{csch} \xi \pm \operatorname{coth} \xi)^{2}$, \\
$u=B+2 A+3 A(\csc \xi \pm \cot \xi)^{2}$, & $u=B-2 A+3 A \tanh \xi /(1 \pm \operatorname{sech} \xi)^{2}$ \\
$u=B+2 A+3 A \sin ^{2} \xi /(1 \pm \cos \xi)^{2}$ & $u$ \\
\hline
\end{tabular}

\section{Demonstrations}

In this section, three solutions of space-time fractional SRLW equation from Table 2 and Table 3 are given. These solutions are demonstrated by the aid of Mathematica 11. 3 .

In all figures, the solutions are investigated for $k=l=1$, and utilizing these constants, $A=-1$ and $B=-2$.

Firstly, let us consider the periodic solution

$$
u=-2-4\left(2 m^{2}-1\right)+12 m^{2} \mathrm{cn}^{2} \xi
$$

in Table 2. 
Figure 1 illustrates this solution for $-5 \leq \xi \leq 5$ and $0 \leq m \leq 1$. Besides, Figure 2 demonstrates 2D graph of the same solution for $-5 \leq \xi \leq 5$ and different $m$ values; namely, the line with dots represents the solution when $m=0$, the unitary line represents the solution when $m=0.5$, the line with dashes represents the solution when $m=0.8$ and the line with dotdashes represents the solution when $m=1$.

Clearly seen from Figure 2 that the wave amplitudes are constant for any chosen $\mathrm{m}$ value but the wave amplitudes and the wavelengths are increasing while $m$ goes from 0 to 1.

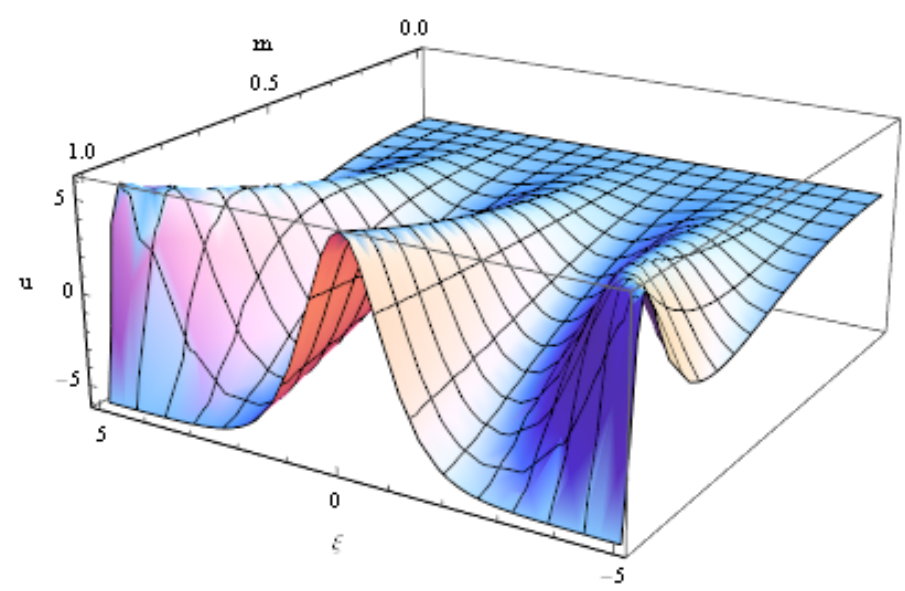

Figure 1. 3D graph of the solution $u(\xi, m)$ when $0 \leq m \leq 1$.

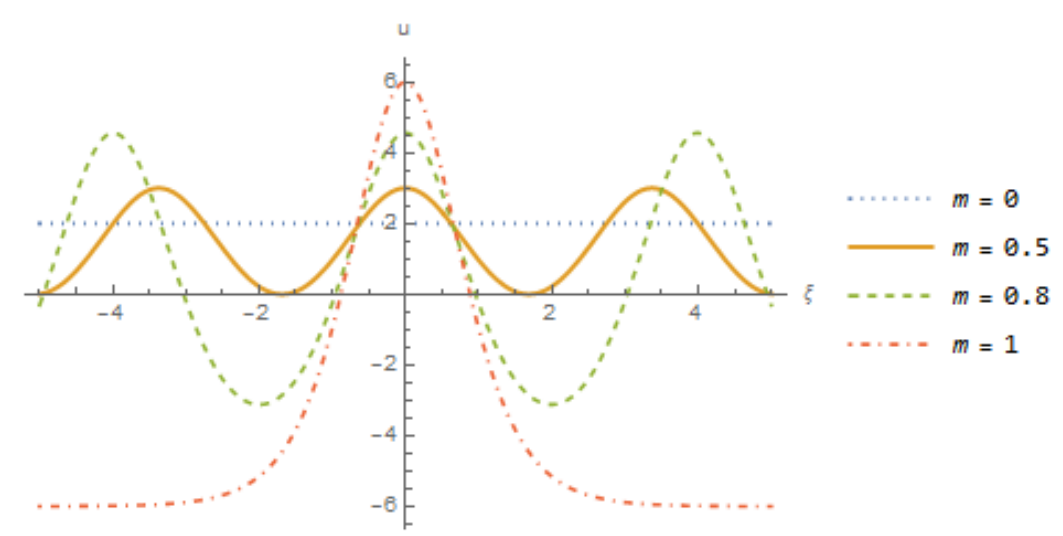

Figure 2. 2D graph of the solution $u(\xi, m)$ when $m=0, m=0.5, m=0.8, m=1$.

Secondly, let us consider space-time fractional SRLW Eq. (1.1) for $\alpha=\beta=0.5$; that is

$$
D_{t}^{1 / 2} D_{t}^{1 / 2} u+D_{x}^{1 / 2} D_{x}^{1 / 2} u+u D_{t}^{1 / 2}\left(D_{x}^{1 / 2} u\right)+\left(D_{t}^{1 / 2} u\right)\left(D_{x}^{1 / 2} u\right)+D_{t}^{1 / 2} D_{t}^{1 / 2}\left(D_{x}^{1 / 2} D_{x}^{1 / 2} u\right)=0 .
$$

Utilizing the transformation $\xi=2 \sqrt{t}+2 \sqrt{x}$, the solution $u=B+8 A+12 A \tan ^{2} \xi$ in Table 3 turns into

$$
u(x, t)=-10-12 \tan ^{2}(2 \sqrt{t}+2 \sqrt{x}) .
$$

Figure 3 demonstrates this solution for $0 \leq x \leq 2$ and $0 \leq t \leq 1$.Besides, Figure 4 illustrates the same solution for $0 \leq x \leq 100$ and $t=1$. Here, the wave amplitudes are goes to infinity, and the wavelengths are increasing when $\mathrm{x}$ increases for $0 \leq x<\infty$.

Finally, let us consider space-time fractional SRLW Eq. (4.1). Using the transformation $\xi=2 \sqrt{t}+2 \sqrt{x}$, the solution $u=B-8 A+12 A \operatorname{coth}^{2} \xi$ in Table 3 turns into

$$
u(x, t)=6-12 \operatorname{coth}^{2}(2 \sqrt{t}+2 \sqrt{x}) .
$$

Figure 5 illustrates this solution for $0 \leq x \leq 4$ and $0 \leq t \leq 1$.Moreover, Figure 6 demonstrates the same solution for $0 \leq x \leq 4$ and $t=1$. 


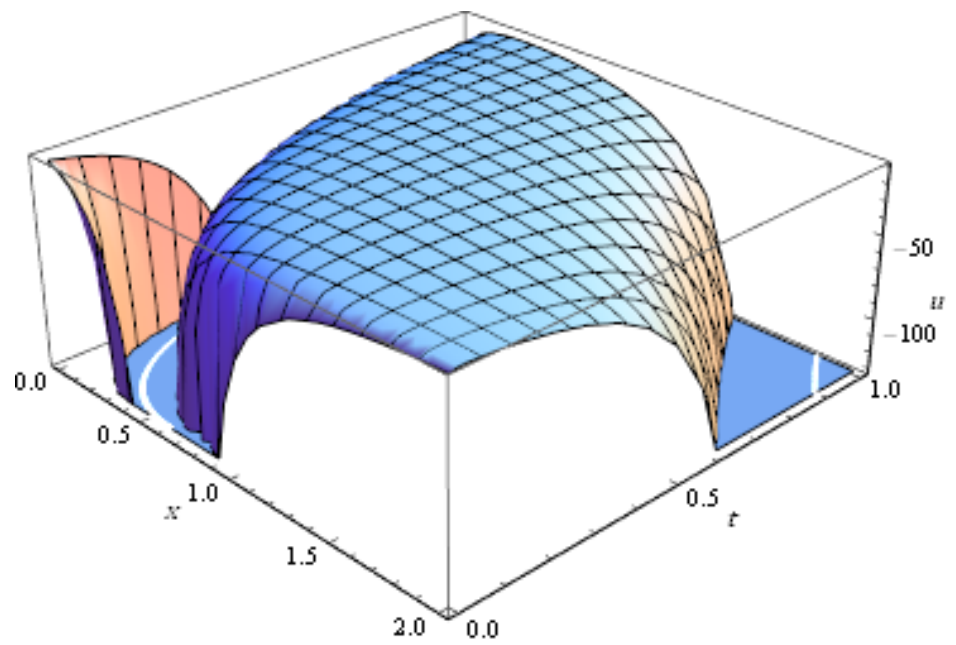

Figure 3. 3D graph of the solution $u(x, t)$ when $m=0$.

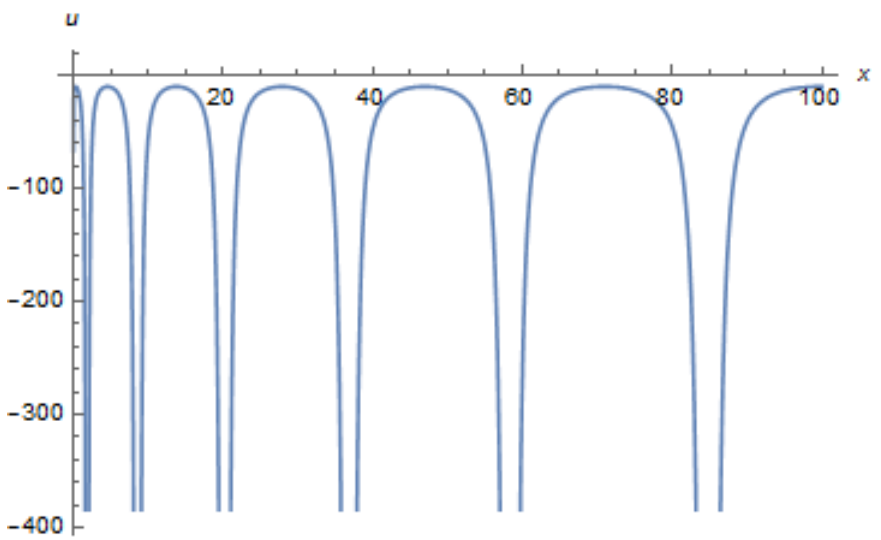

Figure 4. 2D graph of the exact solution $u(x, t)$ when $m=0$ and $t=1$.

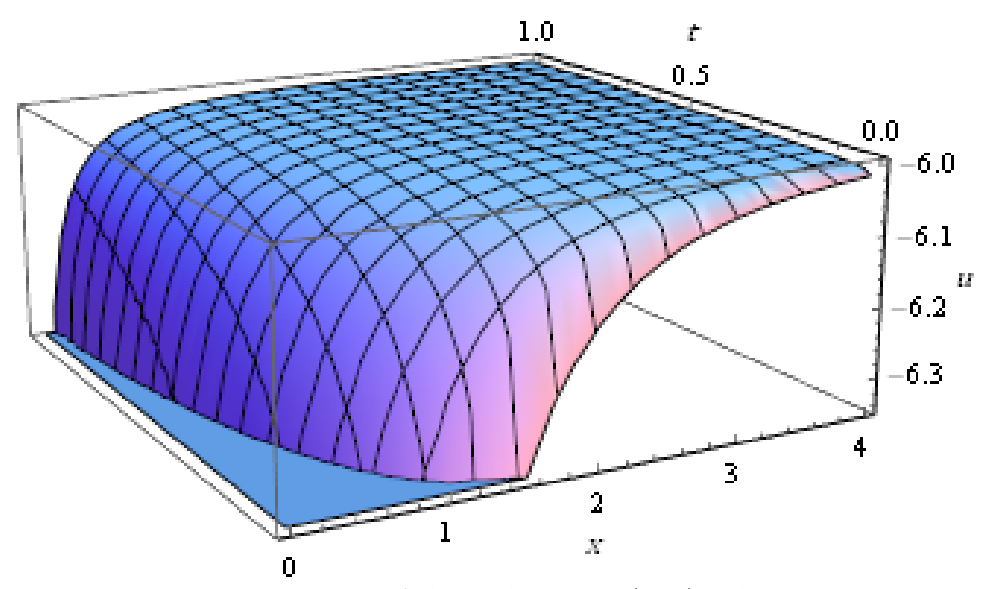

Figure 5. 3D graph of the solution $u(x, t)$ when $m=1$. 


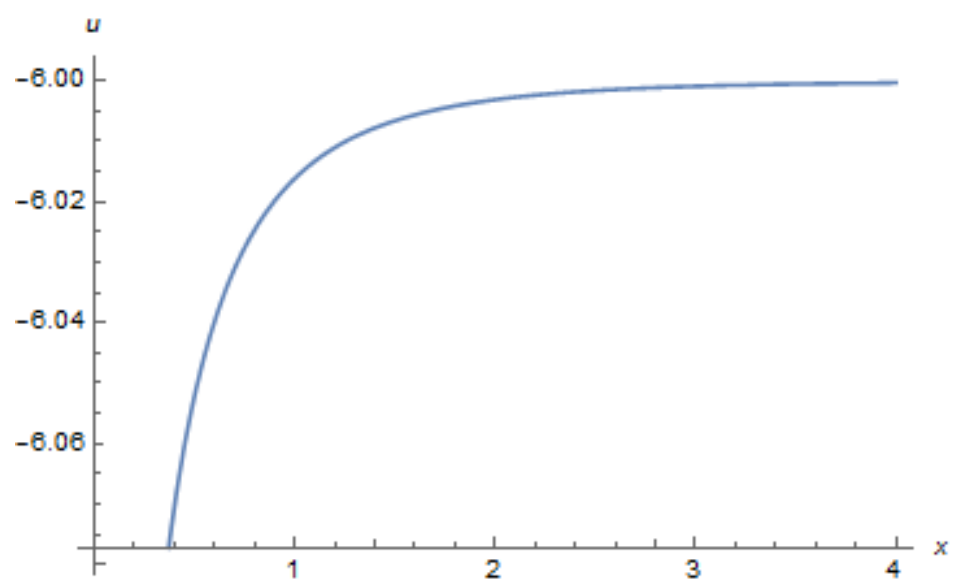

Figure 6. 2D graph of the exact solution $u(x, t)$ when $m=1$.

\section{Conclusions}

In this paper, a direct method based on the Jacobi elliptic functions is presented to gain the exact solutions of space-time fractional SRLW equation. The suggested method has many advantages such that the solutions are found in the form including the rational, trigonometric, hyperbolic functions. The complex valued solutions, soliton solutions, and periodic solutions are also obtained. Some of these solutions are illustrated by two-dimensional and three-dimensional graphics. The other advantage of the proposed method is not to require perturbation, linearization, initial and boundary conditions. Besides, solutions of an auxiliary nonlinear ordinary differential equation have been investigated, and given by table. Solving this auxiliary equation, solutions of Duffing equation are also found. Moreover, using this equation, many partial differential equations can be solved.

In the literature, rational $\left(G^{\prime} / G\right)$-expansion [14], new extended direct algebraic [18], improved Bernoulli subequation function [19], and modified extended tanh [20] methods include the conformable derivatives. 3 solutions which are trigonometric and hyperbolic are obtained by rational $\left(G^{\prime} / G\right)$-expansion method, 37 solutions which are rational, exponential, trigonometric and hyperbolic are obtained by new extended direct algebraic method, 3 solutions which are rational and exponential are obtained by improved Bernoulli sub-equation function method and 12 solutions which are trigonometric and hyperbolic are obtained by modified extended tanh method. Besides, 8 solutions which are trigonometric, hyperbolic and Jacobi elliptic function by the extended Jacobi elliptic function expansion method [16]. When compared with these methods, our method has the largest number of solutions. Because there are 192 type linear independent solutions for 30 different cases in the presented method. The number of the solutions are infinite depending on the parameters $\mathrm{P}, \mathrm{Q}, \mathrm{R}, \mathrm{K}$ and $\mathrm{m}$. When solving different differential equations, the solutions given in Table 1 of auxiliary equation (3.2) can also be utilized. Moreover, these solutions can be helpful for different solution methods. Therefore, these solutions contain the widest set of solutions in the literature.

\section{References}

[1] Seyler, C. E., Fenstermacher, D. L.: A symmetric regularized-long-wave equation. Physics of Fluids. 27 (1), (1984).

[2] Ahmadian, S., Darvishi, M. T.: New exact traveling wave solutions for space-time fractional $(1+1)$-dimensional SRLW equation. Optik. 127, 10697-10704 (2010).

[3] Wang, T., Zhang, L., Chen, F.: Conservative schemes for the symmetric regularized long wave equations. Appl. Math. Comput. 190, 1063-1080 (2007).

[4] Xu, F.: Application of Exp-function method to symmetric regularized long wave (SRLW) equation. Phys. Lett. A. 372, 252-257 (2008).

[5] Abazari, R.: Application of $\left(G^{\prime} / G\right)$-expansion method to traveling wave solutions of three nonlinear evolution equations. Comput. Fluids., 39, 1957-1963 (2010).

[6] Jafari, H., Kadkhoda, N., Khalique, C.M.: Travelling wave solutions of nonlinear evolution equations using the simplest equation method. Comput. Math. Appl., 64, 2084-2088 (2012). 
[7] Hu, J., Zheng, K., Zheng, M.: Numerical simulation and convergence analysis of a high-order conservative difference scheme for SRLW equation. Appl. Math. Modelling., 38, 5573-5581 (2014).

[8] Uğurlu, Y., Kaya, D., İnan, İ. E.: Generalized Jacobi Elliptic Function Method for Periodic Wave Solutions of SRLW Equation and (1+1)-Dimensional Dispersive Long Wave Equation. Çankaya Univ. J. Sci. Eng., 8(2), 205-223 (2011).

[9] Yimnet, S., Wongsaijai, B., Rojsiraphisal, T. and Poochinapan, K.: Numerical implementation for solving the symmetric regularized long wave equation. Appl. Math. Comput. 273, 809-825 (2016).

[10] Alzaidy, J. F.: The fractional sub-equation method and exact analytical solutions for some nonlinear fractional PDEs. Amer. J. Math. Anal. 1 (1), 14-19 (2013).

[11] Guner, O., Eser, D.: Exact solutions of the space time fractional symmetric regularized long wave equation using different methods. Adv. Math. Physics. 2014, Article ID 456804 (2014).

[12] Shakeel M., Mohyud-Din, S. T.: A novel $\left(G^{\prime} / G\right)$-expansion method and its application to the space-time fractional symmetric regularized long wave (SRLW) equation. Adv. Trends Math., 2, 1-16 (2015).

[13] Zhouzheng, K.: Infinite sequence solutions for space-time fractional symmetric regularized long wave equation. J. Partial Differential Equations, 29, 48-58 (2016).

[14] Islam, T., Akbar, M. A., Azad, A. K.: Travelling Wave Solutions to Some Nonlinear Fractional Partial Differential Equations Through the Rational $\left(G^{\prime} / G\right)$-expansion Method. J. Ocean Eng. Sci., 3, 76-81 (2018).

[15] Yaro, D., Seadawy, A. R., Lu, D., Apeanti W. O., Akuamoah, S. W.: Dispersive Wave Solutions of the Nonlinear fractional Zakhorov-Kuznetsov-Benjamin-Bono-Mahony Equation and Fractional Symmetric Regularized Long Wave Equation. Results Phys. 12, 1971-1979 (2019).

[16] Sonmezoğlu, A.: Exact Solutions for Some Fractional Differential Equations. Adv. Math. Phys. 2015, Article ID $567842(2015)$.

[17] Akbulut, A., Kaplan M., Bekir, A.: Auxiliary equation method for fractional differential equations with modified Riemann-Liouville derivative. Int. J. Nonlin. Sci. Num. (2016). DOI: 10.1515/ijnsns-2016-0023.

[18] Senol, M.: New analytical solutions of fractional symmetric regularized-long-wave equation. Rev. Mex. Fis. E, 66(3), 297-307 (2020).

[19] Ala, V., Demirbilek, U., Mamedov, K. R.: An application of improved Bernoulli sub-equation function method to the nonlinear conformable time-fractional SRLW equation. AIMS Math., 5 (4), 3751-3761 (2020).

[20] Ali, K. K., Nuruddeen, R. I., Raslan, K. R.: New structures for the space-time fractional simplified MCH and SRLW equations. Chaos Solitons Fractals. 106, 304-309 (2018).

[21] Jebreen, H. B.: Some Nonlinear Fractional PDEs Involving $\beta$-Derivative by Using Rational exp $(-\Omega(\nu))$-Expansion Method. Complexity. 2020, Article ID 9179826 (2020). https:/ / doi.org/10.1155/2020/9179826

[22] Hanif, M., Habib, M. A.: Exact solitary wave solutions for a system of some nonlinear space-time fractional differential equations. Pramana - J. Phys. 94, 7 (2020).

[23] Manafian, J., Ilhan, O. A., Avazpour, L.: The extended auxiliary equation mapping method to determine novel exact solitary wave solutions of the nonlinear fractional PDEs. (2020). https://doi.org/10.1515/ijnsns-2019-0279.

[24] Alhamdan, W. M., Wazzan, L.: Exact Solutions for the Space-Time Fractional SRLW and STO Equations by the $\left(D^{\alpha} G\right) / G$-Expansion Method. American J. Appl. Math. Stat., 4(3), 87-93 (2016).

[25] Ege, S. M., Misirl1, E.: Traveling wave solutions of some fractional differential equations. Romanian J. Math. Comput. Sci., 6(1), 106-115 (2016).

[26] Zayed, E. M. E., Amer, Y. A., Shohib, R. M. A.: The fractional $\left(D_{\xi}^{\alpha} / G\right)$-expansion method and its applications for solving four nonlinear space-time fractional PDES in mathematical physics. Ital. J. Pure Appl. Math., 34, 463-482 (2015). 
[27] Erdelyi, A., Magnus, W., Oberhettinger, F., Tricomi, F. G.: Higher Transcendental Functions, vol. 2. McGraw-Hill. New York (1953).

[28] Abramowitz, M., Stegun, I. A.: Handbook of Mathematical Functions with Formulas, Graphs, and Mathematical tables. Dover. Newyork (1972).

[29] Khalil, R., Horani, M. A., Yousef, A., Sababheh, M.: A new definition of fractional derivative. J. Comput. Appl. Math. 264, 65-70 (2014).

[30] Abdeljawad, T.: On conformable fractional calculus. J. Comput. Appl. Math. 279, 57-66 (2015).

[31] Kovacic, I., Brennan, M. J.: The Duffing Equation: Nonlinear Oscillators and their Behaviour. John Wiley and Sons. United Kingdom (2011).

[32] Daşcioğlu, A., Çulha, S., Varol Bayram, D.: New analytical solutions of the space fractional KdV equation in terms of Jacobi elliptic functions. New Trends Math. Sci. 5(4), 232-241 (2017).

[33] Çulha S., Daşcioğlu, A.: Analytic solutions of the space-time conformable fractional Klein-Gordon equation in general form. Wave Random Complex 29(4), 775-790 (2019).

[34] Çulha Ünal, S., Daşcioğlu, A., Varol Bayram, D.: New exact solutions of space and time fractional modified Kawahara equation. Phys. A, 551, 124550 (2020).

[35] Daşcioğlu, A., Çulha Ünal, S., Varol Bayram, D.: Neww analytical solutions for space and time Fractional Phi-4 equation. Naturengs, MTU J. Eng. Nat. Sci., 1(1), 30-46 (2020).

[36] Daşcioğlu, A., Çulha Ünal, S.: New exact solutions for the space-time fractional Kawahara equation. Appl. Math. Model., 89, 952-965 (2021).

\section{Affiliations}

SEvil ÇUlha ÜNAL

AdDress: Pamukkale University, Dept. of Mathematics, 20070, Denizli-Turkey.

E-MAIL: sevilclh@gmail.com

ORCID ID: 0000-0001-7447-9219

AYŞEGÜl DAŞCIOĞLU

AdDRESS: Pamukkale University, Dept. of Mathematics, 20070, Denizli-Turkey.

E-MAIL: aakyuz@pau.edu.tr

ORCID ID:0000-0001-8931-6930

DileK VAROL

AdDRESS: Pamukkale University, Dept. of Mathematics, 20070, Denizli-Turkey.

E-MAIL: dvarol@pau.edu.tr

ORCID ID:0000-0002-5158-5614 